\title{
On Mathematics, Imagination and the Beauty of Numbers
}

\section{Citation}

Mazur, Barry C., and Peter Pesic. 2005. On mathematics, imagination \& the beauty of numbers. Daedalus 134(2): 124-130.

\section{Published Version}

doi:10.1162/0011526053887365

\section{Permanent link}

http://nrs.harvard.edu/urn-3:HUL.InstRepos:10356589

\section{Terms of Use}

This article was downloaded from Harvard University's DASH repository, and is made available under the terms and conditions applicable to Other Posted Material, as set forth at http:// nrs.harvard.edu/urn-3:HUL.InstRepos:dash.current.terms-of-use\#LAA

\section{Share Your Story}

The Harvard community has made this article openly available.

Please share how this access benefits you. Submit a story.

Accessibility 


\title{
Dialogue between Barry Mazur \& Peter Pesic
}

\author{
On mathematics, imagination \\ \& the beauty of numbers
}

PETER PESIC: Many intelligent people only see in mathematics a wasteland of dreary formalism, a mind-numbing expanse of theorems and proofs expressed in very abstract language. Doubtless this is partly due to the way it is taught, but such teaching is almost universal, the product of good intentions and much effort. The disconnection between the inner, lived world of mathematicians and the mainstream of intelligent people is very deep, despite the sensual character of mathematics that you describe so well in your recent book, Imagining Num-

Barry Mazur, a Fellow of the American Academy since 1978, is the Gerhard Gade University Professor at Harvard University, where he has taught for over four decades. He has done research in many aspects of pure mathematics and is the author of "Imagining Numbers: (particularly the square root of minus fifteen)" (2003).

Peter Pesic is a tutor and musician-in-residence at St. John's College in Santa Fe, New Mexico. He is the author of "Labyrinth: A Search for the Hidden Meaning of Science" (2000), "Seeing Double: Shared Identities in Physics, Philosophy, and Literature" (2001), "Abel's Proof: An Essay on the Sources and Meaning of Mathematical Unsolvability" (2003), and "Sky in a Bottle" (2005).

(C) 2005 by the American Academy of Arts \& Sciences bers: (particularly the square root of minus fifteen). This raises a hard question: How - if at all - can this living world of mathematics become accessible?

BARRY MAZUR: I can't answer that question, but I can offer some comments. A person's first steps in his or her mathematical development are exceedingly important. Early education deserves our efforts and ingenuity. But also here is a message to any older person who has never given a thought to mathematics or science during their school days or afterwards: You may be ready to start. Starting can be intellectually thrilling, and there are quite a few old classics written in just the right style to accompany you as you begin to take your first steps in mathematics. I'm thinking, for example, of the old T. C. Mits series, or Tobias Dantizg's wonderful Number: The Language of Science, or Lancelot Hogben's Mathematics for the Millions. Moreover, one should not be dismayed that there are many steps there is no need to take them all. Just enjoy each one you do take.

Bill Thurston, a great geometer, uses the word 'tall' to describe mathematics: math is a tall subject in the sense that skyscrapers are tall. That is, one piece of mathematics lies on top of a prior piece of mathematics and lies under the next piece of mathematics, etc. To get to the

Doedalus Spring 2005 
fiftieth story you must traverse all the prior forty-nine, and in the right order. I like this image, but would want to insist that it may be more of a Gaudi-esque structure, with a wide choice of alternate staircases joining and crossing so if you are ever uncomfortable with one route - if the risers are too high, or not high enough - there are other, more accommodating stairwells. And besides, even the view from the first story is a marvel.

PP: What is your earliest memory of mathematics?

$\mathrm{BM}$ : The very earliest was when I was seven or eight years old. My father, who was always fascinated with numbers, would shower me with arithmetical queries like, What is the number that when you double it and add one gives you eleven? I don't think I was particularly adept at finding the answers to these problems, but I did love them. My method was, of course, trial and error. Then, after an especially long barrage of such queries, my father smiled at me and said, "I'll tell you a secret. Here is how you can do these problems more quickly." The "secret" he imparted to me was to invoke the magical X of algebra, restate the problem in the language of algebra, and then to simplify the algebraic sentence, where by simplify he meant solve for X. That X became, after simplification, an actual number, which astonished me. My father also insisted on the ritual palindrome of analysis and synthesis, in the sense that once the value of X was found, I was to redo the steps of the derivation in reverse order to check that the number I came up with for X really worked.

I suppose that I had an especially literal mind, for I actually did think that this information was some sort of secret. A family secret, perhaps, as there might be family secret recipes for particular dishes. I remember being stunned a few years later in a math class, for somehow the teacher had gotten wind of this secret and seemed to be in the process of explaining it to the entire class.

PP: As you confronted this secret, how did it act on you, and especially on your imagination?

BM : I think it acted more on my sense of wonder than on any concrete imaginings.

To work out those simple queries (e.g., What is that number which when you double it and add one you get eleven?) is rather like seeing a concrete visual image develop out of a blank nothing on photographic paper in a darkroom tray. You start with something you deemed X, and at the end of the process you discover $\mathrm{X}$ to be concrete, some particular number. There is a sense of power in this (as you and I know, the early algebraists were very aware of this unexpected power) and in having this power be 'secret' as well. What could be more enticing? When I realized this was part of a much larger common heritage, I wasn't sad: it made it that much larger a clubhouse. My early fascination was that out of pure thought, starting with nothing, something concrete emerges. I remember, a good deal later, being still struck by the equation nothing + thought $=$ something.

About a year before high school, I became an avid reader of popular books about electronics and math. When I was building radio receivers (maladroitly, for the most part) I had the idea of deriving Maxwell's equations by pure thought. How this was going to be accomplished was not so clear, for it is too simpleminded to imagine that some Saint Anselmian strategy (making the sole assumption, for example, that the laws 
governing radio transmission were the "most perfect laws") would lead to laws none other than Maxwell's. But when I was in high school I had complete faith that such a derivation was possible.

PP: In those early days, to what extent was your access to mathematics mediated through physical devices like radios or through visualization, as in electronic schematics? I am thinking of Einstein's insistence that he was always primarily a visual thinker, not an abstract one.

$\mathrm{BM}$ : Let me respond to this question going from the back to the front. I don't think I ever deal with things that are abstract. To be more explicit, I don't like the word 'abstract' except as a comparative term, even though lots of mathematicians use it in a way that reminds me of the dangling comparatives that sometimes show up in ordinary speech. Texts and courses have titles like Abstract Algebra, etc.; my impulse when I see these is to wonder, "Abstract compared to what?" To put it another way, think of the tactic of taking a concept that has arisen in one context and then stripping it from that particular context. For instance, start with Euclidean geometry in the full expression of its geometric intuition and with all its axioms, then strip a few axioms from the list and consider the structure that ensues, with either no concrete realization in mind, or at least as an entity of thought separate from any geometric realization. This is a situation where I believe it is helpful to say that one has abstracted a structure, separating it from its habitual concrete and visualizable context.

Even so, I'm hesitant to use such a Latinate word as 'abstract' for this mode of thought. Aristotle, for example, has at least two different ways of referring to the activity of abstraction: He employs the verb aphairein, which indeed means to 'abstract,' to 'take away.' But at times, as in Book 13 of the Metaphysics, he employs the more explanatory phrase "to take that which does not exist in separation and consider it separately" - a description that has, to my mind, a less scary aspect. But once the concept has been, as people say, abstracted, or once it is, as Aristotle would say, taken separately, if one is to deal effectively with it, one must floodlight it with intuitions of some sort or other. If one is really thinking about this 'abstracted' concept and working with it seriously, it will become utterly as concrete as any other concept. Of course, one may have to homegrow the appropriate intuitions to deal seriously with it.

Electronics, or at least circuitry at the primitive level that I used as a kid, was saturated with concretizing analogies. As I'm sure you know, Kirchhoff's law and Ohm's law are made vivid by a simple analogy to hydraulics - plumbing, if you wish. And Maxwell, when he sought to give vocabulary for the energy in electromagnetic fields, went surprisingly further with this analogy: the somewhat mysterious displacement current that he denoted $j$ (and that seemed so wonderful to me when I first encountered it) - a marvelous concretization of 'action at a distance.'

PP: What you are saying here is consistent with what you write in Imagining Numbers, where you seek felt correlates for an 'abstract' concept like $i=\sqrt{ }-1$. But now you are extending this view in a daring way. What happens then in 'abstract' thinking on the level that you and other mathematicians pursue it, in which (at least for many intelligent people) there seems to be no trace of any sensual, concrete content?
Mathematics, imagination \& the beauty of numbers 
Dialogue between Barry Mazur \& Peter Pesic
BM: I think that analogy is a powerful tool, and it can extend, indefinitely, the range of what we are happy to call concrete, or sensual. Let us start with the truism that the stock-in-trade of poets is to concretize things by analogy. Any snatch of poetry offers some illustration of this. Consider, for example, these lines of Yeats: "Like a long-legged fly upon the stream / His mind moves upon silence." Here the equation is between something that is concrete/sensual and external (the "long-legged fly upon the stream") and something that might actually be even more intimately connected to us, but much harder to catch and hold still: a curious interior state.

Mathematicians are constantly using analogy to expand the realm of what they hold to be concrete. The ubiquitous activity of generalizing, which is one of the staples of mathematical and scientific progress, is a way of analogizing. We start with a structure or concept we feel at home with (say, multiplication of ordinary numbers), and we see a broader realm for which the same or at least an analogous structure or concept may possibly make sense (say, think of composition of transformations as a kind of multiplication operation). We make ourselves at home with this more general concept, initially at least, by depending heavily on the analogy it has with the more familiar, less general concept.

One genre of analogizing in mathematics is to deal with a problem that at first does not seem to be geometric by recasting it in geometric language. For example, consider Fredholm's idea for finding the (unique) function that is the solution of a certain type of equation by translating the problem to that of finding a fixed point of a certain distanceshrinking transformation on a geometric space.
PP: In your view, is there, then, any part of mathematics that is radically divorced from sensual intuition? What about number theory, where there is no geometric, hence visual, field, at least at first glance?

BM: I don't think there is any mathematics radically divorced from some kind of vivid intuition that illuminates it and ties it to the sensual.

You say that number theory has no geometric, hence visual, field at first glance - but that is only at first glance. For most practitioners of number theory these days, number theory is intensely geometric. In the late 1950 and early 1960 I was a geometer, a topologist, and the hook that got me fascinated with number theory was to understand that the set of integers

$$
\ldots-3,-2,-1,0,+1,+2,+3 \ldots
$$

has properties closely analogous to the three-dimensional sphere. Strange as it may seem, the prime numbers are analogous to knots (closed non-self-intersecting loops) in the three-dimensional sphere. Once you see this analogy you begin to see deeply instructive parallels between geometry and number theory.

For example, the skew symmetry of the linking number of one knot relative to another is somehow formally related to what is known in number theory as quadratic reciprocity (a deep reciprocal relationship, initially discovered by Gauss, that holds between any two prime numbers). This is hardly the only analogy that ties number theory to geometry - there are so many that very often it is hard to classify a theorem as being in the one field or the other.

The connections here began as far back as in the works of Abel, in that Galois theory itself sits - ambiguously - between geometry (the study of 
finite coverings of spaces) on the one hand, and algebra (the study of solutions of polynomial equations). This relationship was thoroughly understood by Kronecker and Weber over a hundred years ago. The mathematical discipline of algebraic geometry already expresses the ineluctable joining of these fields.

Sixty years ago, André Weil dreamt up a striking way of very tightly controlling and counting the number of solutions of systems of polynomial equations over finite fields (this being a quintessentially number-theoretic problem) by surmising that there must be a tool for number theory closely analogous to the basic topological theory that efficiently counts the numbers of intersections that one geometric subspace has with another subspace when both are contained within a larger ambient space. All this apparatus has now been set up and establishes a vivid geometric mode of understanding polynomials and systems of polynomials in any algebraic context; indeed, much of number theory is now very comfortably viewed as a piece of a smooth-working synthesis, usually referred to as arithmetic algebraic geometry.

This long-winded answer, then, is simply to say that to many current researchers, number theory is inseparable from geometry, and much mathematical work occurs in a realm that is - marvelously a synthesis of the two.

PP : But what about considerations involving higher dimensions than the three of common spatial experience? Must we rely on analogies to that common world? To what extent would that be possible without, perhaps, deluding ourselves that we are really understanding those more complex spaces, not just squashing them to fit our limited senses?
$\mathrm{BM}$ : But I think we are squashing them, and slicing them, to fit our limited senses - or at least to fit the limits that our senses are constrained to at present. And squashing them is a prelude to understanding them. Without some real innovation, real insight, and exercise of imagination, you don't even know how to begin any squashing procedure.

Squash how? Any act of squashing takes work, and the work itself is what expands one's intuition - expands the limits of our senses. Let me remind you of some standard examples. The most immediate source of examples does not come from high dimensions, but is already in our three-dimensional space of common experience. To visualize things well in three dimensions takes some artifice. Think of the repertoire: the top view, side view, front view, etc., of architectural drawings; the Mercator and other projections to render the globe flat; the CAT scans and MRIs that make pictures of slices of three-dimensional bodies, these slice pictures being taken in various moving and rotating planes and then cleverly put together to render a more faithful understanding of the full three-dimensionality of the examined body. Or think, if you wish, about that chair you are looking at, which you have only one view of (give or take a bit of the parallax of your two eyes and your moving head), and whose utter three-dimensionality you are so at home with.

In a way, all the artifices, as I called them, which work so well for us to substantiate our common three-dimensional experience, are there to be employed to bring higher dimensions into our ken as well. The special theory of relativity deals with four coordinates $(x, y, z, t)$ usually referred to as 'space-time,' and the usual way of thinking graphically about anything happening in this fourdimensional geometry is as a movie of

\section{Mathematics, imagination \& the beauty of numbers}


Dialogue between Barry Mazur \& Peter Pesic three-dimensional slices changing in time.

But there are other modes of squashing the thing down to our limited senses, thereby, in effect, extending those senses. For example, one might envision the four-dimensional space as a planar (i.e., two-parameter) family of planes that, taken all together, fill out fourdimensional space: every point in fourdimensional space will lie on exactly one of these two-dimensional slices. The fun here is that you need a two-dimensional collection of these two-dimensional slices to sweep out the entirety of fourdimensional space: $2+2=4$, after all. You then have the option of thinking of (or visualizing, if you wish) any geometric object in four-dimensional space in terms of how it is diced by this procedure. This type of intuition is very well developed in people who do complex analysis.

Even this list understates the issue. One isn't quite finished if I just give you a finite repertoire - a bag of tricks, so to speak, in the art of squashing - because at a point in one's development of these intuitions, one actually sees more than the mere sum of tricks. One realizes that there is a certain unexpected pliability of spatial intuitions that makes spaces of any dimension equally accessible equally accessible, and in certain respects (and here's a surprise) more easily accessible than lower-dimensional spaces. Topologists understand very well that for certain important work, higherdimensional spaces are simply easier than lower-dimensional spaces - there's more room to move around!

For example, the Poincaré conjecture was first proved by Steve Smale in the mid-196os in dimensions equal to five or more. It took well over a decade after that for it to be proved by Michael Friedman in dimension four. Dimension three is still open, although a Russian mathematician, Perelman, has recently announced that he has a proof. The short answer here is that one will always try to reach out as far as one can with whatever intuition one has and squash as much as one can into it. Doing this squashing has the effect of extending and improving our intuition.

PP: Are there no spaces that are utterly alien to our intuition, only available through a kind of reasoning that is not accessible to our senses?

BM: I want to think of our intuition as not an inert, unchanging resource, but rather as something that can expand when challenged, when exercised. And the mechanism that forces this expansion is analogy. So, are there spaces utterly alien to our intuition? All I can say is that I don't think utterly.

PP: But the very struggle of human imagination to extend itself so far past its common limits indicates that these spaces really may transcend our sensibility. We struggle to grasp new mathematical truths not just because it is hard to visualize them, but also because they defy our most deeply held presumptions. For instance, we try to visualize the infinite-dimensional Hilbert space of quantum theory using visual analogues, but a spinning ball is utterly unlike an electron with spin. At a certain point, doesn't the visual and anthropomorphic fail just because we have gone beyond what we can visualize? And doesn't symbolic mathematics then save us by allowing us to reason securely even when we can no longer see?

BM: What you say is unassailable. But the full panoply of our mathematical intuitions - the intuitions that mathe- 
matics helps strengthen, and refines - is not limited to pure visualization or pure symbolic combinatorial processes or, for that matter, pure any one thing.

I would say that the most powerful of our intuitions are combinations: a potent blending of visualization and artful algebra, of intrinsic canniness of estimation, and of all the intuitions that are the children of sheer experience - knowing when to approximate, when to insist on exact calculations, when to neglect some terms, when to pay the closest attention to them, when to rely upon an analogy, when to distrust it, and ... well, I would not want to limit this list.

But there are two things I would like to emphasize about the ingredients of the brew I just described. The first is that these intuitions tend to amplify, to magnify, each other. The second is that these intuitions show up and are, in some form, perfectly available to anyone who tries their hand at understanding any piece of mathematics, however elementary.

Now let's return to your example of the infinite-dimensional Hilbert space that provides a model for quantum mechanical considerations. I think the notion of infinite-dimensional Hilbert space is a wonderful example of how algebra amplifies the range of visualizability of geometry. A Hilbert space is, almost by formal definition, a space, of never mind how many dimensions, such that any two-dimensional plane in it has all the properties of the Euclidean plane. You can think of it as being very, very visualizable in two-dimensional slices despite its immensity, along with a guarantee from Hilbert that this very feature of it - visualizability in slices - is what is going to be most relevant.

Now once we (or initially, I suppose, Hilbert) hit upon this idea, our basic intuitions regarding Euclidean geome- try - the Euclidean geometry of our high school days - become magically available even in contexts where we would hardly have dared to imagine that visualization would have any relevance.

The example you offer of electrons with spin modeled in terms of Hilbert space is a great testimonial, precisely, to the manner in which visualization as an intuition can be amplified and made more powerful by mathematical analogies. Our comprehension of Euclidean geometry is amplified, thanks to Hilbert, to be a useful thing in understanding even the most seemingly unvisual aspects of atomic particles.

PP: Here you point to new possibilities that would surprise many people who consider themselves mathematically hopeless. Perhaps they think themselves incapable of abstraction or manipulating formalism. You are telling them that, on the contrary, it is the sensual side they are missing.

BM: Living mathematics is in no way abstract, at least to the people who live it. Intuitions can tie mathematics to the most concrete pictures, sensual experiences, and things that are immediate to all of us. There are always loads of alternate routes. If you are blocked at one route, no problem - try another. I believe this is the common understanding of just about everyone who practices mathematics. Mathematics is often taught without such connections, but there is no reason that it can't be taught so that a student's intuitions are fully engaged and exercised every step of the way.
Mathematics, imagination \& the beauty of numbers 